\title{
La unidad de diálisis, ¿un entorno accesible?
}

\author{
Antonio López González ${ }^{1,2,3}$ - Lorena Díaz Rodríguez ${ }^{1,2,3}$ - Carmen Ornosa Agra ${ }^{1}$
}

${ }^{1}$ Diplomado/a en Enfermería

${ }^{2}$ Máster en Gestión e Investigación en la discapacidad y en la dependencia

${ }^{3}$ Máster en Intervención en la discapacidad y en la dependencia

Unidad de Hemodiálisis. U.S.P. Hospital Santa Teresa. A Coruña

\section{Resumen}

Uno de los efectos producidos por los notables cambios demográficos experimentados en las últimas décadas en nuestro país es el aumento de las personas con discapacidad. Ante esta situación, los gobiernos han ido implantando políticas sociales y sanitarias con la finalidad de garantizar el derecho a la igualdad. En este aspecto cobra especial relevancia, como elemento de calidad de vida y de integración, el derecho a la accesibilidad universal entendida esta como "la condición que deben cumplir los entornos, procesos, bienes, productos y servicios, así como los objetos o instrumentos, herramientas y dispositivos, para ser comprensibles, utilizables y practicables por todas las personas en condiciones de seguridad y comodidad y de la forma más autónoma y natural posible".

El objetivo de este estudio es analizar la situación de accesibilidad de las instalaciones de la Unidad de Diálisis del USP Hospital Santa Teresa, proponer tipos de mejora para la adaptación del entorno, conocer la percepción del profesional acerca de la accesibilidad de la Unidad y analizar el grado de conocimiento e implicación relacionado con los derechos de las personas con discapacidad y adultos mayores.

Correspondencia:

Antonio López González

U.S.P. Hospital Santa Teresa (Unidad de Hemodiálisis)

C. / Londres, n². (15009) A Coruña / España

E-mail: antonlopezglez@hotmail.com
PALABRAS CLAVE:

- DISCAPACIDAD

- ACCESIBILIDAD

- UNIDAD DE DIÁLISIS

\section{The dialysis unit, an accessible environment?}

\section{Abstract}

One of the effects of the considerable demographic changes which have come about in recent decades in Spain is the increase in the number of people with disabilities. Faced with this situation, governments have implemented social and healthcare policies with the aim of guaranteeing the right to equality. In this context the right to universal accessibility taken to mean "the condition which must be met by environments, processes, goods, products and services, as well as objects or instruments, tools and devices, for them to be understandable, usable and practicable for everybody in conditions of safety and convenience and in the most autonomous, natural way possible" is especially relevant as a factor in quality of life and integration.

The object of this study is to analyse the situation as regards accessibility of the facilities in the Dialysis Unit of the USP Hospital Santa Teresa, to propose improvements to the environment, to find out 
healthcare professionals' perception of accessibility in the Unit and to assess the level of knowledge and commitment in relation to the rights of disabled and elderly people.

\section{KEY WORDS:}

\section{- DISABILITY}

ACCESSIBILITY

- DIALYSIS UNIT

\section{Introducción}

Los cambios demográficos experimentados en las últimas décadas en España han traído consigo profundas transformaciones en la pirámide poblacional, entre ellas un proceso de envejecimiento notable. Uno de los posibles efectos es el aumento de las personas con discapacidad, ya que la edad es un factor determinante en la aparición de este fenómeno. En España existen alrededor de 3,85 millones de personas discapacitadas, de las cuales más del $65 \%$ son personas mayores de 65 años. La mayor tasa de discapacidad se da en Galicia (112,9 por mil habitantes), seguida de Extremadura (109,9 por mil habitantes). El grupo de deficiencia más frecuente es el de las articulaciones y huesos que causa discapacidad a más de 1,2 millones de personas ${ }^{1}$.

Una de las discriminaciones que más afecta a este colectivo es la falta de accesibilidad entendiendo esta, como "la condición que deben cumplir los entornos, procesos, bienes, productos y servicios, así como los objetos o instrumentos, herramientas y dispositivos, para ser comprensibles, utilizables y practicables por todas las personas en condiciones de seguridad y comodidad y de la forma más autónoma y natural posible". Por culpa de las barreras (urbanísticas, arquitectónicas, de transporte, de comunicación), diversas situaciones de la vida cotidiana, que para muchos son simples y sencillas, se tornan imposibles de sobrellevar lo que vulnera el principio de igualdad y los derechos de las personas con discapacidad y adultos mayores. Sin embargo, la accesibilidad no sólo es un derecho de las personas que tienen discapacidades permanentes o edad avanzada

sino que es necesaria también para las personas con discapacidades temporales como lesiones, fracturas 0 daños pasajeros a la vista; también para las mujeres embarazadas o con niños pequeños ${ }^{2}$.

Desde los años 70 el aumento en la concienciación pública sobre la importancia de la accesibilidad como elemento de calidad de vida y de integración de las personas con discapacidad ha provocado que los principales organismos internacionales se replantearan las políticas relativas a la discapacidad. Esta nueva ética de la discapacidad dio lugar al actual marco legal donde confluyen distintos ordenamientos jurídicos (internacionales, de la Unión Europea, estatales y de cada Comunidad Autónoma) con el objetivo de definir un marco jurídico global ${ }^{3}$.

En España, aunque con anterioridad se hayan desarrollado órdenes y decretos que contienen criterios sobre accesibilidad (tales como las destinadas a las viviendas para minusválidos, elevadores, etc.), la promulgación de la Ley 13/1982, de integración social de los minusválidos (LISMI) significó la primera definición del amparo especial que la Constitución Española reconoce, fundamentalmente en sus artículos 9.2 y 14, a las personas con discapacidad. Posteriormente, la continua evolución socio-política del concepto de discapacidad dieron lugar al nacimiento de la Ley $51 / 2003$, de igualdad de oportunidades, no discriminación y accesibilidad universal de las personas con discapacidad y posteriormente y la Ley 39/2006, de promoción de la autonomía personal y atención a las personas en situación de dependencia. La Ley 51/2003 destaca con la introducción del principio de accesibilidad universal, mientras que la Ley 39/2006 representa una fuerte apuesta por la eliminación de las barreras que garanticen un acceso igualitario y la participación plena ${ }^{4}$.

En materia de accesibilidad, las comunidades autónomas tienen competencia exclusiva, y han optado en su mayoría por la elaboración de leyes que contienen principios generales, objetivos y definiciones, relegando a rango reglamentario disposiciones en forma de normas técnicas que determinan cómo debe entenderse la accesibilidad en los diferentes espacios. En Galicia, el referente legal es la Ley $8 / 1997$ de accesibilidad y supresión de barreras en la Comunidad autónoma de Galicia ${ }^{5}$, desarrollado por el Decreto 35/2000 de 28 de Enero de $2000^{6}$. 
Dada la importancia y necesidad de aumentar el nivel de accesibilidad de los espacios, este estudio responde a cuatro objetivos generales: analizar la situación de accesibilidad de las instalaciones de la Unidad de Diálisis del USP Hospital Santa Teresa y su entorno inmediato, proponer tipos de mejora para la adaptación del entorno, conocer la percepción del profesional acerca de la accesibilidad de la Unidad de Diálisis y, por último, analizar el grado de conocimiento e implicación del personal de enfermería relacionado con los derechos de las personas con discapacidad y adultos mayores.

\section{Material y métodos}

Se ha llevado a cabo un estudio descriptivo transversal. El objeto a estudio ha sido las instalaciones de la unidad de diálisis del USP Hospital Santa Teresa de A Coruña.

En primer lugar, se ha realizado una búsqueda bibliográfica para profundizar en aspectos como la legislación sobre accesibilidad para personas con discapacidad. También, se han analizado las bases de datos estadísticos de carácter general para conocer el número y tipo de discapacidades presentes en Galicia y en el resto del territorio nacional.

Con el fin de apoyar la justificación de este estudio, se ha analizado el tipo de discapacidad presente (física, sensorial, cognitiva) en los pacientes en diálisis en nuestra unidad, utilizado la entrevista y la revisión exhaustiva de sus historias clínicas.

Para la valoración de la accesibilidad de la unidad, se han elaborado 57 ítems que siguen las directrices de la Ley 8/1997 de accesibilidad y supresión de barreras en la Comunidad Autónoma de Galicia y de las recomendaciones de la Sociedad Española de Nefrología 7 . Estos ítems nos proporcionarán parámetros que nos permitirán evaluar las siguientes estancias de la unidad: estacionamiento y ruta de acceso, comunicación horizontal, servicios higiénicos, vestuarios y sala de hemodiálisis.

Por último, se ha prestado atención en conocer la percepción del personal de enfermería implicado en el cuidado del paciente renal. Se ha valorado su grado de conocimiento e implicación con este colectivo y se les ha propuesto que evalúen la accesibilidad de la unidad.
La recopilación de los datos ha sido realizada en 0ctubre de 2011. Los datos se han organizado en tablas. Las variables cualitativas se presentan con cifras absolutas y porcentajes con su correspondiente intervalo de confianza al $95 \%$. Las variables cuantitativas se presentan como una media y desviación estándar.

\section{Resultados}

Hasta el período de estudio, se ha prestado servicio a un total de 94 pacientes con una edad media de 66 $+13,70$, siendo el 58,51\% (IC 95\% 47,88-68,58\%) de los pacientes adultos mayores. El 70,21\% (IC 95\% 59,90-79,21\%) presentan algún tipo de discapacidad, destacando la motora presente en el $29,79 \%$ (IC $95 \%$ $20,79-40,1 \%$ ) necesitando ayuda técnica para su desplazamiento un $17,02 \%$ (IC $95 \%$ 10,05-26,16\%).

En relación a la accesibilidad de las instalaciones, la unidad consta de aparcamiento y acceso independiente exclusivo para pacientes en hemodiálisis. El aparcamiento tiene una superficie de $311 \mathrm{~m}^{2}$ con una capacidad aproximada de 9 vehículos y zona para ambulancias. El itinerario de acceso al interior de la unidad está contiguo al aparcamiento a una distancia aproximada de 20 metros, sin necesidad de la utilización de escaleras 0 ascensores. El acceso al interior se realiza a través de una puerta de cristal de doble hoja (tabla 1).

Estacionamiento independiente para pacientes en hemodiálisis

Estacionamiento adecuado y señalizado para discapacitados

Ruta de acceso a la instalación que evite el uso de escaleras

Ancho de la ruta de acceso es de al menos 0,90 metros

Señalización de las rutas de acceso accesibles a la instalación

Pavimento antideslizante en el estacionamiento y en la ruta de acceso

Puerta de entrada con ancho libre de al menos 0,80 metros

Manija de la puerta: altura de 0,90-1,20 metros

Manijas de la puerta: operativas con el puño cerrado

Apertura de la puerta sin ejercer demasiada fuerza

Presencia de color contraste entre el marco y la puerta

Tabla 1. Estacionamiento y ruta de acceso 
El acceso a las distintas zonas o locales de la unidad (salas de hemodiálisis, almacén, aseos, vestuarios) se realiza a través de un pasillo principal de $31,3 \mathrm{~m}^{2}$ con superficie antideslizante terminado en una puerta de características idénticas a la puerta principal que separa a la unidad del resto de departamentos del hospital (tabla 2).

\begin{tabular}{l|l|}
\hline $\begin{array}{l}\text { La entrada principal provee de acceso libre a la unidad } \\
\text { de hemodiálisis }\end{array}$ & $\boldsymbol{V}$ \\
\hline $\begin{array}{l}\text { Espacio que permita el giro de una silla de ruedas } \\
\text { (ancho } 1,50 \mathrm{~m} \text { ) y después de la puerta }\end{array}$ & $\boldsymbol{V}$ \\
\hline El ancho del pasillo principal es de almenos 1,80 metros & $\boldsymbol{V}$ \\
\hline $\begin{array}{l}\text { Estrechamientos puntuales del pasillo principal no menores } \\
\text { de } 0,90 \text { metros }\end{array}$ & $\boldsymbol{V}$ \\
\hline $\begin{array}{l}\text { Existe señalización conductiva y accesible para personas } \\
\text { discapacitadas }\end{array}$ & $\mathbf{X}$ \\
\hline $\begin{array}{l}\text { Todos los pasillos comunicantes tienen un ancho mínimo de } \\
0,90 \text { metros }\end{array}$ & $\boldsymbol{V}$ \\
\hline $\begin{array}{l}\text { Cambios de dirección: espacio libre que permita el giro de una } \\
\text { silla de ruedas (ancho } 1,50 \text { m) }\end{array}$ & $\boldsymbol{V}$ \\
\hline Existe señalización accesible de la Salida de Emergencias & $\mathbf{X}$ \\
\hline
\end{tabular}

Tabla 2. Circulación horizontal
La unidad de hemodiálisis está dotada 3 aseos para uso exclusivo de pacientes en hemodiálisis: Aseo 1 de 4,71 $\mathrm{m}^{2}$ mixto, Aseo 2 de 2,96 $\mathrm{m}^{2}$ para uso de mujeres y Aseo 3 de $3 \mathrm{~m}^{2}$ para hombres (tabla 3 ).

Contiguo a los aseos 2 y 3 se localizan dos vestuarios de uso exclusivo para pacientes en hemodiálisis con acceso directo a la unidad: uno para mujeres (Vestuario 1 ) de $4,04 \mathrm{~m}^{2}$ y otro para los hombres (Vestuario 2) (tabla 4).

La instalación dispone de dos salas para la realización de las sesiones de hemodiálisis Una primera sala con una superficie de $112 \mathrm{~m}^{2}$ con dos puntos de acceso cada uno con su respectiva puerta y pasillo distribuidor, que dispone de 17 puestos de hemodiálisis. Una segunda sala de $25,56 \mathrm{~m}^{2}$ con un solo acceso y tres puestos de hemodiálisis destinados a pacientes que por motivos clínicos requieren aislamiento. (Tabla 5).

Por otra parte, el 84,37\% (IC 95\% 67,21-94,72\%) del personal que integra el equipo de enfermería de diálisis está completamente seguro de que, actualmente, la falta de accesibilidad es un problema social

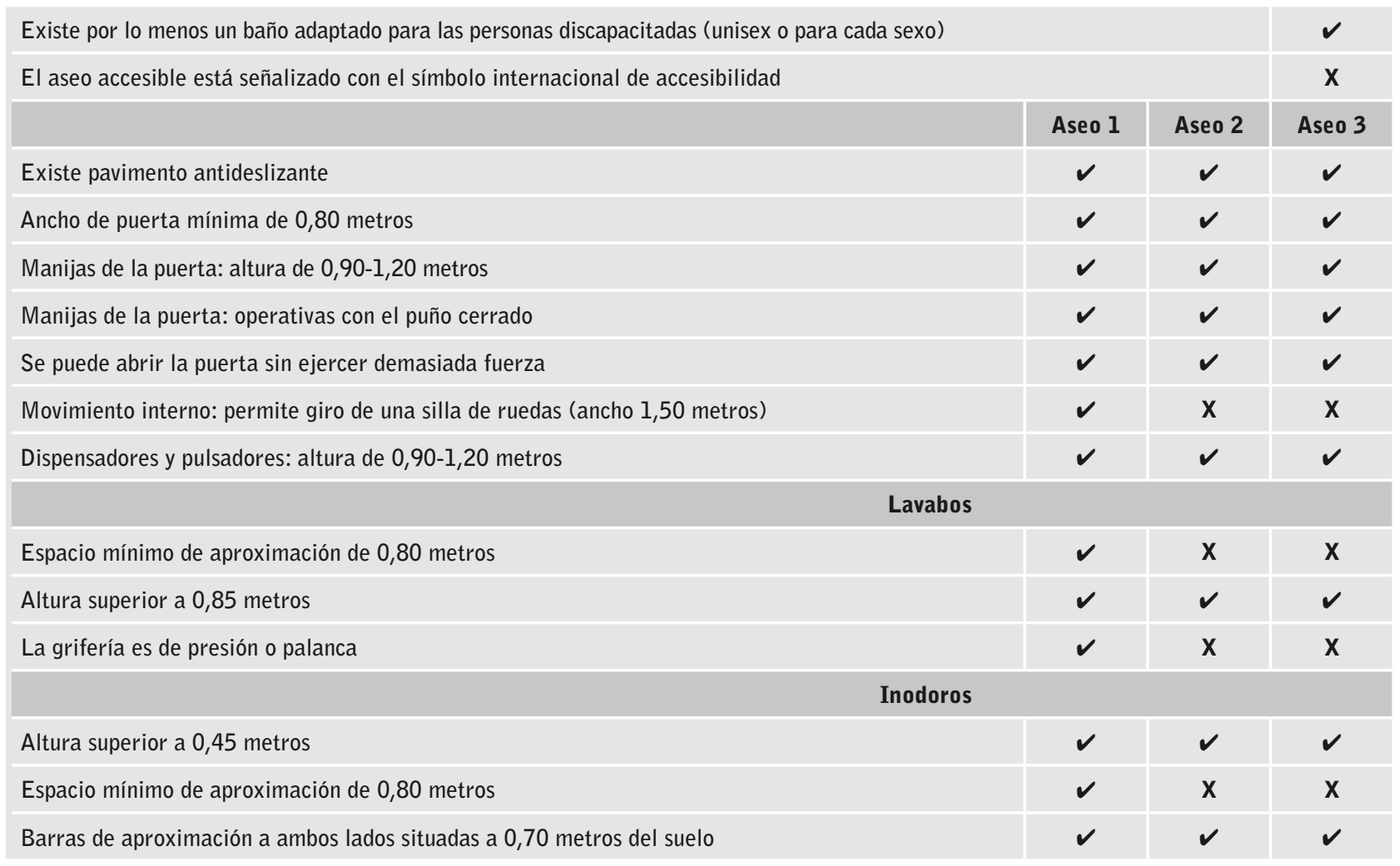

Tabla 3. Servicios Higiénicos 


\begin{tabular}{|l|c|c|}
\hline & Vestuario $\mathbf{1}$ & Vestuario 2 \\
\hline Superficie mínima $1,80 \times 1,70$ metros & $\mathbf{X}$ \\
\hline Pavimento antideslizante & $\mathbf{X}$ \\
\hline Aproximación lateral al mobiliario: dimensión mínima 0,80 metros & $\checkmark$ \\
\hline Percheros situados a una altura comprendida entre $0,90-1,20$ metros & $\mathbf{X}$ \\
\hline Movimiento interno: permite giro de la silla de ruedas (ancho 1,50 metros) & $\checkmark$ \\
\hline Existe asiento adosado a la pared: altura 0,45 metros & $\mathbf{V}$ \\
\hline
\end{tabular}

Tabla 4. Vestuarios

\begin{tabular}{|c|c|c|c|}
\hline & \multicolumn{2}{|c|}{ Vestuario 1} & Vestuario 2 \\
\hline Pavimento antideslizante & \multicolumn{2}{|c|}{$\checkmark$} & $\checkmark$ \\
\hline \multirow[t]{2}{*}{ Altura de los interruptores de la luz $0,90-1,20$ metros } & \multicolumn{2}{|c|}{$\checkmark$} & $\checkmark$ \\
\hline & Puerta 1 & Puerta 2 & \\
\hline La puerta tiene un ancho libre de al menos 0,80 metros & $\checkmark$ & $\checkmark$ & $\checkmark$ \\
\hline Manijas de la puerta: altura de $0,90-1,20$ metros & $\checkmark$ & $\checkmark$ & $\checkmark$ \\
\hline Manijas de la puerta: operativas con el puño cerrado & $\checkmark$ & $\checkmark$ & $\checkmark$ \\
\hline Se puede abrir la puerta sin ejercer demasiada fuerza & $\checkmark$ & $\checkmark$ & $\checkmark$ \\
\hline Los pasillos de acceso permiten el paso de camillas & $\checkmark$ & $\checkmark$ & $\checkmark$ \\
\hline Movimiento interno: permite el giro de sillas de ruedas (ancho $1,50 \mathrm{~m}$ ) & \multicolumn{2}{|c|}{$\mathbf{x}$} & $\mathbf{x}$ \\
\hline Zonas que permiten el giro de camillas (ancho $2,40 \mathrm{~m}$ ) & \multicolumn{2}{|c|}{$\checkmark$} & $\mathbf{x}$ \\
\hline $\mathrm{N}^{0}$ de puestos con superficie mínima de $8 \mathrm{~m}^{2}$ & \multicolumn{2}{|c|}{$14 / 17$} & $3 / 3$ \\
\hline Sillones automatizados & \multicolumn{2}{|c|}{$\checkmark$} & $\checkmark$ \\
\hline Altura de los sillones a 0,45 metros & \multicolumn{2}{|c|}{$\checkmark$} & $\checkmark$ \\
\hline $\mathrm{N}^{0}$ de puestos que permiten una aproximación lateral de $0,80 \mathrm{~m}$ & \multicolumn{2}{|c|}{$16 / 17$} & $3 / 3$ \\
\hline
\end{tabular}

Tabla 5. Sala de hemodiálisis

importante. El 59,38\% (IC 95\% 40,64-76,30\%) del personal no es conocedor del marco jurídico que regula este derecho. Además, el 61,54\% (IC 95\% 31,58$84,14 \%$ ) del personal del equipo de enfermería de diálisis considera que la unidad está acondicionada para garantizar el derecho de accesibilidad. Sin embargo, los aseos están considerados por un $84,61 \%$ (IC $95 \%$ $54,55-90,07 \%$ ) de los entrevistados como el espacio menos accesible.

\section{Discusión}

La accesibilidad universal surge impulsada por la influencia de una sociedad dinámica, moderna y pluralista con el objetivo claro de mejorar la calidad de vida de todas las personas con especial mención a las personas con discapacidad y adultos mayores. Sin embargo, el conseguir un entorno accesible no es tarea sencilla ya que son muchas las variables que entran en juego (económicas, falta de formación, de sensibilización, de voluntad política, etc.) y, se hará más complejo aún, sin un verdadero compromiso social.

El trabajo de observación de la instalación llevado a cabo, nos muestra que a pesar de ser una instalación antigua las distintas remodelaciones acaecidas en los últimos años han proporcionado a la unidad un alto grado de accesibilidad arquitectónica. Sin embargo, se podrían realizar mejoras en distintas estancias 
que permitan alcanzar el estatus de accesibilidad universal:

- Habilitar una plaza de parking dedicada exclusivamente a usuarios discapacitados con las características y señalización adecuada según la Ley vigente.

- Sustitución de las puertas de apertura manual de acceso a la unidad (acceso desde el exterior y acceso desde el interior) por puertas con apertura automática.

- Unificación de baños y vestuarios (Aseo 2 y 3 Vestuario 1 y 2) con el fin de ganar espacio que permita la reubicación de elementos y sustitución de dispositivos accesibles (grifería, etc.)

- Reestructurar los puestos de hemodiálisis con la finalidad de ganar amplitud tanto lateral como frontal y reubicar el mobiliario para liberar espacio que permita el giro tanto de silla de ruedas como de camas.

- Señalizar todos los elementos o rutas accesibles como tales utilizando los símbolos internacionales de accesibilidad recogidos en la legislación.

Por otra parte, se ha demostrado que el personal integrante del equipo de enfermería responsable del cuidado del paciente renal en nuestro hospital está implicado y sensibilizado con la problemática de la accesibilidad de las personas discapacitadas y adultos mayores, a pesar del escaso grado de conocimiento acerca del marco jurídico que regula este derecho. Esto viene a evidenciar que la profesión de enfermería, además de sus sólidos conocimientos científicos y técnicos, tiene un carácter esencialmente humano justificado por su capacidad empática. Sin embargo, se propone la realización de sesiones formativas que actualicen y refuercen los conocimientos acerca de este colectivo para optimizar nuestros cuidados.

Para finalizar, este estudio pone de manifiesto que la tarea de adecuar entornos no es exclusiva de colectivos profesionales como los arquitectos o ingenieros, sino que la implicación de múltiples disciplinas, como la enfermería, posibilitan la optimización de las intervenciones ya que permite ahondar en la problemática desde una óptica distinta. También, el influjo de otras disciplinas en nuestro trabajo enfermero nos va a permitir iden- tificar de un modo más exhaustivo las necesidades de este colectivo y asentar de manera firme las bases de una intervención integral que persiga la mejora de la calidad de vida de los pacientes discapacitados y adultos mayores.

Recibido: 10 Enero 2012

Revisado: 30 Enero 2012

Modificado: 12 Febrero 2012

Aceptado: 20 Febrero 2012

\section{Bibliográficas}

1. Instituto Nacional de Estadística. Encuesta de Discapacidad, Autonomía personal y situaciones de Dependencia (EDAD) [en línea]. 2008. www.ine. es [acceso: Octubre 2011].

2. De Asís R., Campoy Cervera I, Bengoechea M. ${ }^{a}$ A. "Derecho a la igualdad y a la diferencia. Análisis de los principios de no discriminación, diversidad y acción positiva", en R. de Lorenzo y L. Cayo Pérez Bueno (dir.), Tratado sobre discapacidad, Thomson. Aranzadi, Cizur Menor, 2007, 115-141

3. Campoy Cervera I., "Reflexiones acerca de los derechos de las personas con discapacidad", en I. Campoy Cervera (ed.), Los derechos de las personas con discapacidad. Perspectivas sociales, políticas, jurídicas y filosóficas, Dykinson. Instituto de derechos humanos "Bartolomé de las Casas". Universidad Carlos III de Madrid, Madrid, 2004, 7-27.

4. Alcaín Martínez E, González-Badía Fraga J, Molina Fernández C. (coord.), Régimen jurídico de las personas con discapacidad en España y la Unión Europea, Comares, Granada, 2006.

5. Accesibilidad y supresión de barreras en la Comunidad Autónoma de Galicia. Ley 8/1997 de 20 de Agosto. Diario Oficial de Galicia, $N^{\circ} 166$, (29/08/1997).

6. Decreto $35 / 2000$, de 28 de enero, por el que se aprueba el Reglamento de desarrollo y ejecución 
de la Ley de accesibilidad y supresión de barreras en la Comunidad de Galicia. Diario Oficial de Galicia, Nº42, (29/02/2000).
7. Fernández Fuentes $A$, Martín de Francisco A.L., Otero González A, Martí i Monros A., Solozábal Campos C., González Parra E.,et al. Guías SEN. Guías de centros de hemodiálisis. Nefrología 2006; 26 Supl $8: 5-10$. 